\title{
Spotlight on Neoadjuvant Therapy for Breast Cancer
}

\author{
Judy C. Boughey, $\mathrm{MD}^{1}$ and Kelly K. Hunt, $\mathrm{MD}^{2}$ \\ ${ }^{1}$ Mayo Clinic, Rochester, MN; ${ }^{2}$ The University of Texas MD Anderson Cancer Center, Houston, TX
}

Historically, the use of neoadjuvant therapy was considered only for patients with locally advanced or inflammatory breast cancer. Increasingly, systemic therapy is being given prior to surgery in patients with operable and early-stage breast cancer. Clinical trials have demonstrated that the use of neoadjuvant systemic therapy is equivalent to adjuvant therapy in terms of overall survival, and it increases the likelihood of successful breast-conserving therapy. As a result, patients who, at the time of presentation, are identified as meeting the criteria for receipt of adjuvant chemotherapy are being considered for delivery of the chemotherapy in the neoadjuvant setting. In addition to reduction in the size of the primary tumor, patients receiving neoadjuvant chemotherapy are less likely to have positive axillary nodes and may be candidates for node-

Acknowledgment: This educational review series, "Neoadjuvant Therapy in Breast Cancer" is supported by an educational grant from Genentech, Inc. The Society of Surgical Oncology offers CME/MOC for this educational review series. Visit moc.surgonc.org for additional information.

Annals of Surgical Oncology educational reviews represent the journal's commitment to the peer review and publication of high quality research necessary to define the safety, toxicity, or effectiveness of potential therapeutic agents compared with conventional alternatives.

This Educational Review Series may include information regarding the use of medications that may be outside the approved labeling for these products. Physicians should consult the current prescribing information for these products. Authors of Annals of Surgical Oncology educational reviews are provided at the time of article solicitation with this statement regarding off-label pharmaceutical information and research.

(C) Society of Surgical Oncology 2015

First Received: 4 February 2015;

Published Online: 2 March 2015

K. K. Hunt, MD

e-mail: khunt@mdanderson.org sparing surgery. The pathologic assessment of the surgical specimens after neoadjuvant therapy provides information on the residual cancer burden (RCB) which correlates with disease-free survival outcomes. Neoadjuvant systemic therapy is no longer limited to chemotherapy but can include targeted agents and endocrine therapies specific for biologic subtypes. The neoadjuvant approach requires a multidisciplinary team, and communication across the disciplines is key to achieving optimal outcomes. In this special issue of Annals of Surgical Oncology is a series of articles focusing on the assessment and management of breast cancer patients treated with systemic therapy in the neoadjuvant setting.

In the first article, Drs. Haddad and Goetz present an overview of the changing landscape of neoadjuvant therapy for breast cancer. ${ }^{1}$ They review what has been learned from landmark trials in the field and how the approach has changed with the availability of targeted agents. When the treatment is targeted to the specific biologic subtype, the likelihood of response to therapy is significantly increased. They provide an overview of the RCB index and the CPS + EG scoring system, which are clinically available prognostic tools that incorporate response to therapy in addition to other clinical and pathologic factors. Finally, they discuss current approaches and options for systemic treatment of patients with newly diagnosed breast cancer based on receptor status (e.g. human epidermal growth factor receptor 2, estrogen receptor, and triple receptor negative).

Understanding the extent of disease in the breast and the regional nodes prior to initiating systemic therapy is critical when utilizing the neoadjuvant approach. In the second article, Dialani et al. present a review of conventional breast imaging with mammography and ultrasound, and how they are used before therapy for staging and after systemic therapy to evaluate response. ${ }^{2}$ Finally, although it may not be ready for routine use, they discuss how functional imaging might be tailored to individual patients in the future based on biologic subtypes. 
Dr. Mamounas discusses the impact of neoadjuvant chemotherapy on the surgical management of breast cancer patients. ${ }^{3}$ Neoadjuvant chemotherapy has been shown to convert patients from requiring mastectomy to candidates for breast-conserving surgery; however, initial studies suggested a higher rate of local-regional recurrence. In this article, he provides pretreatment, post-treatment, and intraoperative guidelines for the physician to ensure the greatest success for targeting all residual disease and for minimizing local-regional recurrence. He discusses the role for the use of sentinel lymph node surgery in patients receiving neoadjuvant chemotherapy and the controversy regarding the timing of sentinel node surgery. Finally, he reviews the recently reported trials on sentinel node surgery after chemotherapy in patients who present with biopsy-proven nodal metastasis. With more developments in targeted therapy, he envisions that options for surgical procedures will vary with response to neoadjuvant therapy, moving towards a more individualized surgical treatment-extent of surgical resection based on how the individual patient's tumor responded to the systemic therapy received.

Garg and Buchholz follow the surgical management with a discussion on the indications for adjuvant radiation therapy in women treated with neoadjuvant chemotherapy. ${ }^{4}$ Traditionally, radiation therapy has been recommended following neoadjuvant chemotherapy to all patients who present with stage III disease regardless of response. Patients with stage II disease can present a challenge to the radiation oncologist, especially in the setting of initial node-positive disease followed by pathologic complete response to systemic therapy. This remains an area of controversy, especially when reconstruction is performed, and requires close communication between the breast surgeon, plastic surgeon, and radiation oncologist. This review summarizes the current status of the indications for adjuvant radiation in patients treated with neoadjuvant chemotherapy, and highlights ongoing clinical trials.

In the final manuscript, Cortazar and Geyer describe the phenomenon of pathologic complete response and association with long-term outcomes. ${ }^{5}$ They review the published data on pathologic complete response and eradication of disease as a result of neoadjuvant chemotherapy, and discuss the pooled analysis that was performed by the US FDA in collaboration with other investigators. Within this context, the authors discuss the decision by the FDA to accept pathologic complete response as a surrogate endpoint for the application for accelerated approval of drugs in the neoadjuvant setting.
The pathologic assessment of the breast and nodal specimens from surgical resection after neoadjuvant chemotherapy is a critical component of care and requires close collaboration and communication with the pathologist. Tissue processing has not been standardized across institutions, or even across clinical trials, which can make it challenging to assess response across populations. The RCB index provides a template for the assessment and documentation of the extent of residual disease within the tumor bed and regional nodes. ${ }^{6} \mathrm{RCB}$ is increasingly utilized to standardize pathologic reporting across sites, and is the reporting mechanism used in the ISPY trials and several ongoing cooperative group trials. The RCB calculator incorporates the size of the residual tumor bed in two dimensions along with the percent tumor cellularity averaged across the whole tumor bed, in addition to the size of the largest lymph node metastasis. Together, these data can be inserted into the RCB index calculator online to determine the RCB class (0, I, II, or III) and also the RCB index (continuous variable). RCB 0 is the same as a pathologic complete response-complete eradication of invasive disease from the breast and the axillary lymph nodes. The higher the RCB, the greater the extent of residual disease and poorer overall outcome. Symmans and colleagues have made the clinical calculator available online, as well as providing a brief video for pathologists who are not familiar with the technique. ${ }^{6}$

It is our hope that this series of articles provides the most contemporary information available regarding the ongoing advances in the management of patients being treated with neoadjuvant systemic therapy. It should serve as a resource for clinicians and patients, and will hopefully foster collaboration and multidisciplinary care.

\section{REFERENCES}

1. Haddad TC, Goetz MP. Landscape of neoadjuvant therapy for breast cancer. Ann Surg Oncol. doi:10.1245/s10434-015-4405-7.

2. Dialani V, Chadashvilli T, Slanetz PJ. Role of imaging in neoadjuvant therapy for breast cancer. Ann Surg Oncol. doi:10. 1245/s10434-015-4403-9.

3. Mamounas EP. Impact of neoadjuvant chemotherapy on localregional surgical treatment of breast cancer. Ann Surg Oncol. doi:10.1245/s10434-015-4406-6.

4. Garg AK, Buchholz TA. Influence of neoadjuvant chemotherapy on radiotherapy for breast cancer. Ann Surg Oncol. doi:10.1245/ s10434-015-4402-x.

5. Cortazar P, Geyer CE. Pathological complete response in neoadjuvant treatment of breast cancer. Ann Surg Oncol. doi:10.1245/ s10434-015-4404-8.

6. Symmans WF, Peintinger F, Hatzis et al. Measurement of residual breast cancer burden to predict survival after neoadjuvant chemotherapy. J Clin Oncol. 2007;25:4414-4422. 